\title{
Boundedness of the Solutions to Certain Delay Dynamic Integrodifferential Systems on Time Scales
}

\author{
Min Fan $\mathbb{D D}^{1}$ and Yazhou Tian $(\mathbb{D})^{2}$ \\ ${ }^{1}$ Qingdao University of Technology, Feixian, Shandong 273400, China \\ ${ }^{2}$ School of Mathematical Sciences, University of Jinan, Jinan, Shandong 250022, China \\ Correspondence should be addressed to Yazhou Tian; tianyazhou369@163.com
}

Received 27 September 2018; Accepted 28 November 2018; Published 9 December 2018

Academic Editor: Allan C. Peterson

Copyright (C) 2018 Min Fan and Yazhou Tian. This is an open access article distributed under the Creative Commons Attribution License, which permits unrestricted use, distribution, and reproduction in any medium, provided the original work is properly cited.

In this paper, the bounds on the solutions of certain delay dynamic integrodifferential systems on time scales are considered. Based on a new Gronwall-Bellman type delay integral inequality, we can estimate the boundedness of solutions to integrodifferential systems. At the end, an example is presented to state the main results.

\section{Introduction}

The theory of time scales was established and developed by Hilger [1] and Bohner and Peterson [2,3]. At present, different kinds of integral inequalities and their applications in differential, integral, and integrodifferential equations have become the research focus; see the papers [4-23]. To list a few, Ma and Pečarić [10] established an integral inequality on time scales to study the boundedness of solutions of the delay dynamic differential system. Wang and $\mathrm{Xu}$ [13] investigated some integral inequalities in two independent variables on time scales. In $[15,16], \mathrm{Ma}$ et al. considered the generalized two-dimensional fractional differential system with Hadamard derivative. However, to the best of our knowledge, there are very little known results on discussing the bounds on the solutions of delay integrodifferential system on time scales.

Motivated by the works in $[10,15,16]$, we further investigate the delay dynamic integrodifferential system on time scales. By introducing a new Gronwall-Bellman type integral inequality, we obtain the bounds on the solutions of a class of delay dynamic integrodifferential system on time scales.

In the following, $\mathbb{R}$ denotes the set of real numbers and $\mathbb{R}_{+}=[0,+\infty), C(M, S)$ represents the class of all continuous functions defined on set $M$ with range in the set $S$. T denotes an arbitrary time scale, $\mathbb{T}_{0}=\left[t_{0},+\infty\right) \cap \mathbb{T}, t_{0} \in \mathbb{T}$, and $C_{r d}$ denotes the set of rd-continuous functions.

\section{Problem Description and Preliminaries}

Consider the following delay integrodifferential system

$$
\begin{gathered}
\left(x^{p}(t)\right)^{\Delta}=G_{1}\left(t, x\left(\tau_{1}(t)\right), y\left(\tau_{1}(t)\right),\right. \\
\left.\int_{t_{0}}^{t} H_{1}\left(t, s, x\left(\tau_{2}(s)\right), y\left(\tau_{2}(s)\right)\right) \Delta s\right), \\
\left(y^{q}(t)\right)^{\Delta}=G_{2}\left(t, x\left(\tau_{1}(t)\right), y\left(\tau_{1}(t)\right),\right. \\
\left.\int_{t_{0}}^{t} H_{2}\left(t, s, x\left(\tau_{2}(s)\right), y\left(\tau_{2}(s)\right)\right) \Delta s\right),
\end{gathered}
$$

$t \in \mathbb{T}_{0}$

with the initial condition

$$
\begin{aligned}
& x(t)=\varphi(t), \\
& y(t)=\psi(t)
\end{aligned}
$$


with $\left|\varphi\left(\tau_{i}(t)\right)\right| \leq\left|C_{1}\right|^{1 / p}$,

$$
\begin{aligned}
& \left|\psi\left(\tau_{i}(t)\right)\right| \leq\left|C_{2}\right|^{1 / q}, \\
& \quad \text { for every } t \in \mathbb{T}_{0} \text { with } \tau_{i}(t) \leq t_{0} \quad(i=1,2),
\end{aligned}
$$

where $G_{i}: \mathbb{T}_{0} \times \mathbb{R}^{3} \longrightarrow \mathbb{R}, H_{i}: \mathbb{T}_{0} \times \mathbb{T}_{0} \times \mathbb{R}^{2} \longrightarrow \mathbb{R}$ are continuous functions, $\tau_{i}: \mathbb{T}_{0} \longrightarrow \mathbb{T}, \tau_{i}(t) \leq t(i=1,2),-\infty<$ $\alpha=\min _{t \in \mathbb{T}_{0}}\left\{\inf \tau_{1}(t), \inf \tau_{2}(t)\right\} \leq t_{0}, C_{1}=x^{p}\left(t_{0}\right), C_{2}=$ $y^{q}\left(t_{0}\right), p, q$ are some positive constants, and $\varphi, \psi \in$ $C_{r d}\left(\left[\alpha, t_{0}\right] \cap \mathbb{T}, \mathbb{R}\right)$.

Remark 1. If we let $H_{i} \equiv 0$, the system considered in this paper reduces to the one in [10].

The following lemmas are useful in our main results.

Lemma 2 (see [4], Lemma 2.1). Assume that $c \geq 0$ and $k \geq$ $l>0$. Then

$$
c^{l / k} \leq \frac{l}{k} m^{(l-k) / k} c+\frac{k-l}{k} m^{l / k}
$$

for any $m>0$.

Lemma 3. Assume that $u, a, b, c \in C_{r d}\left(\mathbb{T}_{0}, \mathbb{R}_{+}\right), d \in$ $C_{r d}\left(\mathbb{T}_{0}^{2}, \mathbb{R}_{+}\right)$, and $a(t)$ is nondecreasing. If

$$
\begin{aligned}
u(t) \leq & a(t)+\int_{t_{0}}^{t} b(s) u(s) \Delta s \\
& +\int_{t_{0}}^{t} c(s)\left(\int_{t_{0}}^{s} d(s, \xi) u(\xi) \Delta \xi\right) \Delta s, \quad t \in \mathbb{T}_{0},
\end{aligned}
$$

then

$$
u(t) \leq a(t) e_{f}\left(t, t_{0}\right), \quad t \in \mathbb{T}_{0}
$$

where

$$
f(t)=b(t)+c(t) \int_{t_{0}}^{t} d(t, \xi) \Delta \xi
$$

Proof. First we assume that $a(t)>0$; from (3), we have

$$
\begin{aligned}
& \frac{u(t)}{a(t)} \\
& \quad \leq 1 \\
& \quad+\int_{t_{0}}^{t}\left(b(s) \frac{u(s)}{a(s)}+c(s) \int_{t_{0}}^{s} d(s, \xi) \frac{u(\xi)}{a(\xi)} \Delta \xi\right) \Delta s .
\end{aligned}
$$

Defining a function $r(t)$ by right side of (6), we obtain

$$
\begin{aligned}
& \frac{u(t)}{a(t)} \leq r(t), \\
& r\left(t_{0}\right)=1,
\end{aligned}
$$

$r(t)$ is nondecreasing, and

$$
\begin{aligned}
r^{\Delta}(t) & =b(t) \frac{u(t)}{a(t)}+c(t) \int_{t_{0}}^{t} d(t, \xi) \frac{u(\xi)}{a(\xi)} \Delta \xi \\
& \leq\left(b(t)+c(t) \int_{t_{0}}^{t} d(t, \xi) \Delta \xi\right) r(t),
\end{aligned}
$$

which implies that

$$
r(t) \leq e_{f}\left(t, t_{0}\right),
$$

where $f(t)$ is defined as in (5). Combining (7) and (9), we get the required inequality (4).

If $a(t)=0$ for $t \in \mathbb{T}_{0}$, we carry out the above procedure with $a(t)+\varepsilon$ instead of $a(t)$, where $\varepsilon>0$ is an arbitrary small constant, and subsequently pass to the limit as $\varepsilon \longrightarrow 0$ to obtain (4). The proof is complete.

\section{Main Results}

Theorem 4. Assume that

$$
\begin{aligned}
\left|G_{1}(t, x, y, u)\right| \leq & l_{11}(t) N_{11}\left(t,|x|^{r_{11}}\right) \\
& +l_{12}(t) N_{12}\left(t,|y|^{r_{12}}\right) \\
& +l_{13}(t)|u|, \\
\left|G_{2}(t, x, y, u)\right| \leq & l_{21}(t) N_{21}\left(t,|x|^{r_{21}}\right) \\
& +l_{22}(t) N_{22}\left(t,|y|^{r_{22}}\right) \\
& +l_{23}(t)|u|, \\
\left|H_{1}(t, s, x, y)\right| \leq & \omega_{13}(t, s) N_{13}\left(s,|x|^{r_{13}}\right) \\
& +\omega_{14}(t, s) N_{14}\left(s,|y|^{r_{14}}\right), \\
& +\omega_{23}(t, s) N_{23}\left(s,|x|^{r_{23}}\right) \\
& +(t, s) N_{24}\left(s,|y|^{r_{24}}\right),
\end{aligned}
$$

$$
t, s \in \mathbb{T}_{0}
$$

hold, where $l_{i j} \in C_{r d}\left(\mathbb{T}_{0}, \mathbb{R}_{+}\right)(i=1,2, j=1,2,3), \omega_{i j} \in$ $C_{r d}\left(\mathbb{T}_{0} \times \mathbb{T}_{0}, \mathbb{R}_{+}\right)(i=1,2, j=3,4), N_{i j}: \mathbb{T}_{0} \times \mathbb{R}_{+} \longrightarrow \mathbb{R}_{+}$is a continuous function satisfying

$$
0 \leq N_{i j}(t, x)-N_{i j}(t, y) \leq M_{i j}(t, y)(x-y)
$$

for $x \geq y>0$, where $M_{i j}: \mathbb{T}_{0} \times \mathbb{R}_{+} \longrightarrow \mathbb{R}_{+}$is continuous $(i=1,2, j=1,2,3,4), p$ and $q$ are constants, and $r_{i j}$ is a constant with $p \geq r_{i j}>0(i=1,2, j=1,3), q \geq r_{i j}>$ $0(i=1,2, j=2,4)$. Furthermore, suppose that $(x(t), y(t))$ is a solution of system (1) satisfying the initial condition (I). Then, for any constant $m>0$,

$$
\begin{aligned}
&|x(t)| \leq\left(\widetilde{A}(t) e_{K}\left(t, t_{0}\right)\right)^{1 / p}, \\
&|y(t)| \leq\left(\Phi_{2}(t)+h_{2}(t) \widetilde{A}(t) e_{K}\left(t, t_{0}\right)\right)^{1 / q} e_{A}^{1 / q}\left(t, t_{0}\right), \\
& t \in \mathbb{T}_{0},
\end{aligned}
$$


where

$$
\begin{aligned}
& \widetilde{A}(t)=\Phi_{1}(t)+\frac{r_{12}}{q} m^{\left(r_{12}-q\right) / q} \int_{t_{0}}^{t} l_{12}(s) M_{12}\left(s, \frac{q-r_{12}}{q}\right. \\
& \left.\cdot m^{r_{12} / q}\right) \Phi_{2}(s) e_{A}\left(s, t_{0}\right) \Delta s+\frac{r_{14}}{q} \\
& \cdot m^{\left(r_{14}-q\right) / q} \int_{t_{0}}^{t} l_{13}(s) \int_{t_{0}}^{s} \omega_{14}(s, \xi) M_{14}\left(\xi, \frac{q-r_{14}}{q}\right. \\
& \left.\cdot m^{r_{14} / q}\right) \Phi_{2}(\xi) e_{A}\left(\xi, t_{0}\right) \Delta \xi \Delta s \\
& A(t)=\frac{r_{22}}{q} m^{\left(r_{22}-q\right) / q} l_{22}(t) M_{22}\left(t, \frac{q-r_{22}}{q} m^{r_{22} / q}\right) \\
& +\frac{r_{24}}{q} m^{\left(r_{24}-q\right) / q} l_{23}(t) \int_{t_{0}}^{t} \omega_{24}(t, \xi) M_{24}\left(\xi, \frac{q-r_{24}}{q}\right. \\
& \left.\cdot m^{r_{24} / q}\right) \Delta \xi \\
& \Phi_{1}(t)=\left|C_{1}\right|+\int_{t_{0}}^{t} l_{11}(s) N_{11}\left(s, \frac{p-r_{11}}{p} m^{r_{11} / p}\right) \Delta s \\
& +\int_{t_{0}}^{t} l_{12}(s) N_{12}\left(s, \frac{q-r_{12}}{q} m^{r_{12} / q}\right) \Delta s \\
& +\int_{t_{0}}^{t} l_{13}(s) \int_{t_{0}}^{s}\left(\omega_{13}(s, \xi) N_{13}\left(\xi, \frac{p-r_{13}}{p} m^{r_{13} / p}\right)\right. \\
& \left.+\omega_{14}(s, \xi) N_{14}\left(\xi, \frac{q-r_{14}}{q} m^{r_{14} / q}\right)\right) \Delta \xi \Delta s, \\
& \Phi_{2}(t)=\left|C_{2}\right|+\int_{t_{0}}^{t} l_{21}(s) N_{21}\left(s, \frac{p-r_{21}}{p} m^{r_{21}} / p\right) \Delta s \\
& +\int_{t_{0}}^{t} l_{22}(s) N_{22}\left(s, \frac{q-r_{22}}{q} m^{r_{22} / q}\right) \Delta s \\
& +\int_{t_{0}}^{t} l_{23}(s) \int_{t_{0}}^{s}\left(\omega_{23}(s, \xi) N_{23}\left(\xi, \frac{p-r_{23}}{p} m^{r_{23} / p}\right)\right. \\
& \left.+\omega_{24}(s, \xi) N_{24}\left(\xi, \frac{q-r_{24}}{q} m^{r_{24}} / q\right)\right) \Delta \xi \Delta s \\
& K(t)=\frac{r_{11}}{p} m^{\left(r_{11}-p\right) / p} l_{11}(t) M_{11}\left(t, \frac{p-r_{11}}{p} m^{r_{11} / p}\right) \\
& +\frac{r_{12}}{q} m^{\left(r_{12}-q\right) / q} l_{12}(t) M_{12}\left(t, \frac{q-r_{12}}{q} m^{r_{12} / q}\right) h_{2}(t) \\
& \cdot e_{A}\left(t, t_{0}\right)+l_{13}(t) \int_{t_{0}}^{t}\left(\frac{r_{13}}{p} m^{\left(r_{13}-p\right) / p} \omega_{13}(t, \xi)\right. \\
& \cdot M_{13}\left(\xi, \frac{p-r_{13}}{p} m^{r_{13} / p}\right)+\frac{r_{14}}{q} m^{\left(r_{14}-q\right) / q} \omega_{14}(t, \xi) \\
& \left.\cdot M_{14}\left(\xi, \frac{q-r_{14}}{q} m^{r_{14} / q}\right) h_{2}(\xi) e_{A}\left(\xi, t_{0}\right)\right) \Delta \xi,
\end{aligned}
$$

$$
\begin{gathered}
h_{2}(t)=\frac{r_{21}}{p} m^{\left(r_{21}-p\right) / p} \int_{t_{0}}^{t} l_{21}(s) M_{21}\left(s, \frac{p-r_{21}}{p}\right. \\
\left.\cdot m^{r_{21} / p}\right) \Delta s+\frac{r_{23}}{p} m^{\left(r_{23}-p\right) / p} \int_{t_{0}}^{t} l_{23}(s) \\
\cdot \int_{t_{0}}^{s} \omega_{23}(s, \xi) M_{23}\left(\xi, \frac{p-r_{23}}{p} m^{r_{23} / p}\right) \Delta \xi \Delta s .
\end{gathered}
$$

Proof. The solution $(x(t), y(t))$ of system (1) satisfies

$$
\begin{array}{r}
x^{p}(t)=C_{1}+\int_{t_{0}}^{t} G_{1}\left(s, x\left(\tau_{1}(s)\right), y\left(\tau_{1}(s)\right),\right. \\
\left.\int_{t_{0}}^{s} H_{1}\left(s, \xi, x\left(\tau_{2}(\xi)\right), y\left(\tau_{2}(\xi)\right)\right) \Delta \xi\right) \Delta s, \\
y^{q}(t)=C_{2}+\int_{t_{0}}^{t} G_{2}\left(s, x\left(\tau_{1}(s)\right), y\left(\tau_{1}(s)\right),\right. \\
\left.\int_{t_{0}}^{s} H_{2}\left(s, \xi, x\left(\tau_{2}(\xi)\right), y\left(\tau_{2}(\xi)\right)\right) \Delta \xi\right) \Delta s .
\end{array}
$$

Combining (10) and (14), we have

$$
\begin{aligned}
& |x(t)|^{p} \leq\left|C_{1}\right|+\int_{t_{0}}^{t} l_{11}(s) N_{11}\left(s,\left|x\left(\tau_{1}(s)\right)\right|^{r_{11}}\right) \Delta s \\
& +\int_{t_{0}}^{t} l_{12}(s) N_{12}\left(s,\left|y\left(\tau_{1}(s)\right)\right|^{r_{12}}\right) \Delta s+\int_{t_{0}}^{t} l_{13}(s) \\
& \cdot \int_{t_{0}}^{s}\left(\omega_{13}(s, \xi) N_{13}\left(\xi,\left|x\left(\tau_{2}(\xi)\right)\right|^{r_{13}}\right)\right. \\
& \left.+\omega_{14}(s, \xi) N_{14}\left(\xi,\left|y\left(\tau_{2}(\xi)\right)\right|^{r_{14}}\right)\right) \Delta \xi \Delta s, \\
& |y(t)|^{q} \leq\left|C_{2}\right|+\int_{t_{0}}^{t} l_{21}(s) N_{21}\left(s,\left|x\left(\tau_{1}(s)\right)\right|^{r_{21}}\right) \Delta s \\
& +\int_{t_{0}}^{t} l_{22}(s) N_{22}\left(s,\left|y\left(\tau_{1}(s)\right)\right|^{r_{22}}\right) \Delta s+\int_{t_{0}}^{t} l_{23}(s) \\
& \cdot \int_{t_{0}}^{s}\left(\omega_{23}(s, \xi) N_{23}\left(\xi,\left|x\left(\tau_{2}(\xi)\right)\right|^{r_{23}}\right)\right. \\
& \left.\quad+\omega_{24}(s, \xi) N_{24}\left(\xi,\left|y\left(\tau_{2}(\xi)\right)\right|^{r_{24}}\right)\right) \Delta \xi \Delta s .
\end{aligned}
$$

Defining

$$
\begin{aligned}
& z_{1}^{p}(t)=\left|C_{1}\right|+\int_{t_{0}}^{t} l_{11}(s) N_{11}\left(s,\left|x\left(\tau_{1}(s)\right)\right|^{r_{11}}\right) \Delta s \\
& +\int_{t_{0}}^{t} l_{12}(s) N_{12}\left(s,\left|y\left(\tau_{1}(s)\right)\right|^{r_{12}}\right) \Delta s+\int_{t_{0}}^{t} l_{13}(s) \\
& \cdot \int_{t_{0}}^{s}\left(\omega_{13}(s, \xi) N_{13}\left(\xi,\left|x\left(\tau_{2}(\xi)\right)\right|^{r_{13}}\right)\right. \\
& \left.+\omega_{14}(s, \xi) N_{14}\left(\xi,\left|y\left(\tau_{2}(\xi)\right)\right|^{r_{14}}\right)\right) \Delta \xi \Delta s
\end{aligned}
$$


and

$$
\begin{aligned}
& z_{2}^{q}(t)=\left|C_{2}\right|+\int_{t_{0}}^{t} l_{21}(s) N_{21}\left(s,\left|x\left(\tau_{1}(s)\right)\right|^{r_{21}}\right) \Delta s \\
& +\int_{t_{0}}^{t} l_{22}(s) N_{22}\left(s,\left|y\left(\tau_{1}(s)\right)\right|^{r_{22}}\right) \Delta s+\int_{t_{0}}^{t} l_{23}(s) \\
& \cdot \int_{t_{0}}^{s}\left(\omega_{23}(s, \xi) N_{23}\left(\xi,\left|x\left(\tau_{2}(\xi)\right)\right|^{r_{23}}\right)\right. \\
& \left.+\omega_{24}(s, \xi) N_{24}\left(\xi,\left|y\left(\tau_{2}(\xi)\right)\right|^{r_{24}}\right)\right) \Delta \xi \Delta s,
\end{aligned}
$$

we can obtain that $z_{1}(t)$ and $z_{2}(t)$ are nondecreasing, and

$$
\begin{aligned}
& |x(t)| \leq z_{1}(t), \\
& |y(t)| \leq z_{2}(t), \\
& t \in \mathbb{T}_{0} .
\end{aligned}
$$

Case 1: for $t \in \mathbb{T}_{0}$ with $\tau_{i}(t)>t_{0}$, we obtain

$$
\begin{aligned}
& \left|x\left(\tau_{i}(t)\right)\right| \leq z_{1}\left(\tau_{i}(t)\right) \leq z_{1}(t), \\
& \left|y\left(\tau_{i}(t)\right)\right| \leq z_{2}\left(\tau_{i}(t)\right) \leq z_{2}(t) .
\end{aligned}
$$

Case 2: for $t \in \mathbb{T}_{0}$ with $\tau_{i}(t) \leq t_{0}$, by the initial condition (I), we have

$$
\begin{aligned}
& \left|x\left(\tau_{i}(t)\right)\right|=\left|\varphi\left(\tau_{i}(t)\right)\right| \leq\left|C_{1}\right|^{1 / p} \leq z_{1}(t), \\
& \left|y\left(\tau_{i}(t)\right)\right|=\left|\psi\left(\tau_{i}(t)\right)\right| \leq\left|C_{2}\right|^{1 / q} \leq z_{2}(t),
\end{aligned}
$$

$t \in \mathbb{T}_{0}$.

Both (19) and (20) imply that

$$
\begin{aligned}
& \left|x\left(\tau_{i}(t)\right)\right| \leq z_{1}(t), \\
& \left|y\left(\tau_{i}(t)\right)\right| \leq z_{2}(t) \\
& \quad(i=1,2), t \in \mathbb{T}_{0} .
\end{aligned}
$$

This together with (16) and (17) yields

$$
\begin{aligned}
& z_{1}^{p}(t) \leq\left|C_{1}\right|+\int_{t_{0}}^{t} l_{11}(s) N_{11}\left(s, z_{1}^{r_{11}}(s)\right) \Delta s \\
& +\int_{t_{0}}^{t} l_{12}(s) N_{12}\left(s, z_{2}^{r_{12}}(s)\right) \Delta s+\int_{t_{0}}^{t} l_{13}(s) \\
& \quad \cdot \int_{t_{0}}^{s}\left(\omega_{13}(s, \xi) N_{13}\left(\xi, z_{1}^{r_{13}}(\xi)\right)\right. \\
& \left.+\omega_{14}(s, \xi) N_{14}\left(\xi, z_{2}^{r_{14}}(\xi)\right)\right) \Delta \xi \Delta s, \\
& z_{2}^{q}(t) \leq\left|C_{2}\right|+\int_{t_{0}}^{t} l_{21}(s) N_{21}\left(s, z_{1}^{r_{21}}(s)\right) \Delta s+\int_{t_{0}}^{t} l_{22}(s) \\
& \quad \cdot N_{22}\left(s, z_{2}^{r_{22}}(s)\right) \Delta s+\int_{t_{0}}^{t} l_{23}(s) \\
& \quad \cdot \int_{t_{0}}^{s}\left(\omega_{23}(s, \xi) N_{23}\left(\xi, z_{1}^{r_{23}}(\xi)\right)\right. \\
& \left.+\omega_{24}(s, \xi) N_{24}\left(\xi, z_{2}^{r_{24}}(\xi)\right)\right) \Delta \xi \Delta s .
\end{aligned}
$$

Define

$$
\begin{aligned}
& u(t)=\left|C_{1}\right|+\int_{t_{0}}^{t} l_{11}(s) N_{11}\left(s, z_{1}^{r_{11}}(s)\right) \Delta s+\int_{t_{0}}^{t} l_{12}(s) \\
& \cdot N_{12}\left(s, z_{2}^{r_{12}}(s)\right) \Delta s+\int_{t_{0}}^{t} l_{13}(s) \\
& \cdot \int_{t_{0}}^{s}\left(\omega_{13}(s, \xi) N_{13}\left(\xi, z_{1}^{r_{13}}(\xi)\right)\right. \\
& \left.+\omega_{14}(s, \xi) N_{14}\left(\xi, z_{2}^{r_{14}}(\xi)\right)\right) \Delta \xi \Delta s, \\
& v(t)=\left|C_{2}\right|+\int_{t_{0}}^{t} l_{21}(s) N_{21}\left(s, z_{1}^{r_{21}}(s)\right) \Delta s+\int_{t_{0}}^{t} l_{22}(s) \\
& \cdot N_{22}\left(s, z_{2}^{r_{22}}(s)\right) \Delta s+\int_{t_{0}}^{t} l_{23}(s) \\
& \cdot \int_{t_{0}}^{s}\left(\omega_{23}(s, \xi) N_{23}\left(\xi, z_{1}^{r_{23}}(\xi)\right)\right. \\
& \left.+\omega_{24}(s, \xi) N_{24}\left(\xi, z_{2}^{r_{24}}(\xi)\right)\right) \Delta \xi \Delta s .
\end{aligned}
$$

Combining (22) and (23), we have

$$
\begin{aligned}
& z_{1}(t) \leq u^{1 / p}(t), \\
& z_{2}(t) \leq v^{1 / q}(t), \\
& t \in \mathbb{T}_{0} .
\end{aligned}
$$

By Lemma 2 and (24), for any real number $m>0$, we get

$$
\begin{aligned}
& z_{1}^{r_{i j}}(t) \leq \frac{r_{i j}}{p} m^{\left(r_{i j}-p\right) / p} u(t)+\frac{p-r_{i j}}{\mathrm{p}} m^{r_{i j} / p} \\
& \quad(i=1,2, j=1,3)
\end{aligned}
$$

and

$$
\begin{aligned}
& z_{2}^{r_{i j}}(t) \leq \frac{r_{i j}}{q} m^{\left(r_{i j}-q\right) / q} v(t)+\frac{q-r_{i j}}{q} m^{r_{i j} / q} \\
& \quad(i=1,2, j=2,4) .
\end{aligned}
$$

By assumption (11) and the last inequalities, it follows that

$$
\begin{aligned}
& N_{i j}\left(s, z_{1}^{r_{i j}}(s)\right) \\
& \leq \frac{r_{i j}}{p} m^{\left(r_{i j}-p\right) / p} M_{i j}\left(s, \frac{p-r_{i j}}{p} m^{r_{i j} / p}\right) u(s) \\
& \quad+N_{i j}\left(s, \frac{p-r_{i j}}{p} m^{r_{i j} / p}\right) \quad(i=1,2, j=1,3)
\end{aligned}
$$

and

$$
\begin{aligned}
N_{i j}\left(s, z_{2}^{r_{i j}}(s)\right) & \\
\leq & \frac{r_{i j}}{q} m^{\left(r_{i j}-q\right) / q} M_{i j}\left(s, \frac{q-r_{i j}}{q} m^{r_{i j} / q}\right) v(s) \\
& +N_{i j}\left(s, \frac{q-r_{i j}}{q} m^{r_{i j} / q}\right) \quad(i=1,2, j=2,4) .
\end{aligned}
$$


Substituting the last inequalities into (23), we can obtain

$$
\begin{aligned}
& u(t) \leq \Phi_{1}(t)+\frac{r_{11}}{p} m^{\left(r_{11}-p\right) / p} \int_{t_{0}}^{t} l_{11}(s) \\
& \cdot M_{11}\left(s, \frac{p-r_{11}}{p} m^{r_{11} / p}\right) u(s) \Delta s+\frac{r_{12}}{q} \\
& \cdot m^{\left(r_{12}-q\right) / q} \int_{t_{0}}^{t} l_{12}(s) M_{12}\left(s, \frac{q-r_{12}}{q} m^{r_{12} / q}\right) \\
& \cdot v(s) \Delta s+\frac{r_{13}}{p} m^{\left(r_{13}-p\right) / p} \int_{t_{0}}^{t} l_{13}(s) \int_{t_{0}}^{s} \omega_{13}(s, \xi) \\
& \cdot M_{13}\left(\xi, \frac{p-r_{13}}{p} m^{r_{13} / p}\right) u(\xi) \Delta \xi \Delta s+\frac{r_{14}}{q} \\
& \cdot m^{\left(r_{14}-q\right) / q} \int_{t_{0}}^{t} l_{13}(s) \int_{t_{0}}^{s} \omega_{14}(s, \xi) \\
& \cdot M_{14}\left(\xi, \frac{q-r_{14}}{q} m^{r_{14} / q}\right) v(\xi) \Delta \xi \Delta s
\end{aligned}
$$

and

$$
\begin{aligned}
& v(t) \leq \Phi_{2}(t)+\frac{r_{21}}{p} m^{\left(r_{21}-p\right) / p} \int_{t_{0}}^{t} l_{21}(s) \\
& \cdot M_{21}\left(s, \frac{p-r_{21}}{p} m^{r_{21} / p}\right) u(s) \Delta s+\frac{r_{22}}{q} \\
& \cdot m^{\left(r_{22}-q\right) / q} \int_{t_{0}}^{t} l_{22}(s) M_{22}\left(s, \frac{q-r_{22}}{q} m^{r_{22} / q}\right) \\
& \cdot v(s) \Delta s+\frac{r_{23}}{p} m^{\left(r_{23}-p\right) / p} \int_{t_{0}}^{t} l_{23}(s) \int_{t_{0}}^{s} \omega_{23}(s, \xi) \\
& \cdot M_{23}\left(\xi, \frac{p-r_{23}}{p} m^{r_{23} / p}\right) u(\xi) \Delta \xi \Delta s+\frac{r_{24}}{q} \\
& \cdot m^{\left(r_{24}-q\right) / q} \int_{t_{0}}^{t} l_{23}(s) \int_{t_{0}}^{s} \omega_{24}(s, \xi) \\
& \cdot M_{24}\left(\xi, \frac{q-r_{24}}{\mathrm{q}} m^{r_{24} / q}\right) v(\xi) \Delta \xi \Delta s,
\end{aligned}
$$

where $\Phi_{1}(t)$ and $\Phi_{2}(t)$ are defined as in (13).

Let

$$
\begin{gathered}
f_{2}(t)=\Phi_{2}(t)+\frac{r_{21}}{p} m^{\left(r_{21}-p\right) / p} \int_{t_{0}}^{t} l_{21}(s) \\
\cdot M_{21}\left(s, \frac{p-r_{21}}{p} m^{r_{21} / p}\right) u(s) \Delta s+\frac{r_{23}}{p} \\
\cdot m^{\left(r_{23}-p\right) / p} \int_{t_{0}}^{t} l_{23}(s) \int_{t_{0}}^{s} \omega_{23}(s, \xi) \\
\cdot M_{23}\left(\xi, \frac{p-r_{23}}{p} m^{r_{23} / p}\right) u(\xi) \Delta \xi \Delta s .
\end{gathered}
$$

From (30), we have

$$
\begin{gathered}
v(t) \leq f_{2}(t)+\frac{r_{22}}{q} m^{\left(r_{22}-q\right) / q} \int_{t_{0}}^{t} l_{22}(s) \\
\cdot M_{22}\left(s, \frac{q-r_{22}}{q} m^{r_{22} / q}\right) v(s) \Delta s+\frac{r_{24}}{q} \\
\cdot m^{\left(r_{24}-q\right) / q} \int_{t_{0}}^{t} l_{23}(s) \int_{t_{0}}^{s} \omega_{24}(s, \xi) \\
\cdot M_{24}\left(\xi, \frac{q-r_{24}}{q} m^{r_{24} / q}\right) v(\xi) \Delta \xi \Delta s
\end{gathered}
$$

Since $f_{2}(t)$ is nondecreasing, by Lemma 3 , we have

$$
v(t) \leq f_{2}(t) e_{A}\left(t, t_{0}\right)
$$

where $A(t)$ is defined as in (13). Since $u(t)$ is nondecreasing, it follows from (33) that

$$
v(t) \leq\left(\Phi_{2}(t)+h_{2}(t) u(t)\right) e_{A}\left(t, t_{0}\right),
$$

where $h_{2}(t)$ is defined as in (13). Substituting (34) into (29), we can obtain

$$
\begin{aligned}
u(t) & \leq \widetilde{A}(t)+\int_{t_{0}}^{t}\left(\frac{r_{11}}{p} m^{\left(r_{11}-p\right) / p} l_{11}(s)\right. \\
\cdot & M_{11}\left(s, \frac{p-r_{11}}{p} m^{r_{11} / p}\right)+\frac{r_{12}}{q} m^{\left(r_{12}-q\right) / q} l_{12}(s) \\
\cdot & \left.M_{12}\left(s, \frac{q-r_{12}}{q} m^{r_{12} / q}\right) h_{2}(s) e_{A}\left(s, t_{0}\right)\right) u(s) \Delta s \\
+ & \int_{t_{0}}^{t} l_{13}(s) \int_{t_{0}}^{s}\left(\frac{r_{13}}{p} m^{\left(r_{13}-p\right) / p} \omega_{13}(s, \xi)\right. \\
\cdot & M_{13}\left(\xi, \frac{p-r_{13}}{p} m^{r_{13} / p}\right)+\frac{r_{14}}{q} m^{\left(r_{14}-q\right) / q} \omega_{14}(s, \xi) \\
& \left.\cdot M_{14}\left(\xi, \frac{q-r_{14}}{q} m^{r_{14} / q}\right) h_{2}(\xi) e_{A}\left(\xi, t_{0}\right)\right) u(\xi) \\
& \cdot \Delta \xi \Delta s .
\end{aligned}
$$

Using Lemma 3, we get

$$
u(t) \leq \widetilde{A}(t) e_{K}\left(t, t_{0}\right)
$$

where $\widetilde{A}(t)$ and $K(t)$ are defined as in (13). From (34), we have

$$
v(t) \leq\left(\Phi_{2}(t)+h_{2}(t) \widetilde{A}(t) e_{K}\left(t, t_{0}\right)\right) e_{A}\left(t, t_{0}\right) .
$$

Combining (36) and (37) with (24) and (18), we obtain the desired inequality (12). This completes the proof.

Remark 5. Theorem 4 generalizes [10, Theorem 3.1].

Remark 6. Assumptions (10) and (11) are easily satisfied. For example, we can choose $N(t, x)=t^{2} x^{1 / 3}$, and it is not difficult to verify that 


$$
\begin{aligned}
0 & \leq N(t, x)-N(t, y)=t^{2}\left(x^{1 / 3}-y^{1 / 3}\right) \\
& \leq M(t, y)(x-y),
\end{aligned}
$$

where $M(t, y)=t^{2} y^{-2 / 3} / 3$.

\section{Application}

In this section, we present an example to illustrate the main results.

Example 7. Consider system (1) with the initial condition (I) and $p=q=2, G_{1}, G_{2}, H_{1}$, and $H_{2}$ satisfy

$$
\begin{aligned}
&\left|G_{1}(t, x, y, u)\right| \leq l_{11}(t)|x|^{1 / 3}+l_{12}(t)|y|^{1 / 2} \\
&+l_{13}(t)|u|, \\
&\left|G_{2}(t, x, y, u)\right| \leq l_{21}(t)|x|^{1 / 3}+l_{22}(t)|y|^{1 / 2} \\
&+l_{23}(t)|u|, \\
&\left|H_{1}(t, s, x, y)\right| \leq \omega_{13}(t, s)|x|^{1 / 2}+\omega_{14}(t, s)|y|^{1 / 4}, \\
&\left|H_{2}(t, s, x, y)\right| \leq \omega_{23}(t, s)|x|^{1 / 2}+\omega_{24}(t, s)|y|^{1 / 4}, \\
& t, s \in \mathbb{T}_{0}
\end{aligned}
$$

with $r_{i j}=1, i=1,2, j=1,2,3,4$. Then the solution of system (1) satisfies

$$
\begin{aligned}
&|x(t)| \leq\left(\widetilde{A}(t) e_{K}\left(t, t_{0}\right)\right)^{1 / 2}, \\
&|y(t)| \leq\left(\Phi_{2}(t)+h_{2}(t) \widetilde{A}(t) e_{K}\left(t, t_{0}\right)\right)^{1 / 2} e_{A}^{1 / 2}\left(t, t_{0}\right), \\
& t \in \mathbb{T}_{0},
\end{aligned}
$$

where

$$
\begin{aligned}
& \widetilde{A}(t)=\Phi_{1}(t)+2^{-3 / 2} m^{-1 / 4} \int_{t_{0}}^{t} l_{12}(s) \Phi_{2}(s) \\
& \cdot e_{A}\left(s, t_{0}\right) \Delta s+2^{-5 / 4} m^{-3 / 8} \int_{t_{0}}^{t} l_{13}(s) \\
& \cdot \int_{t_{0}}^{s} \omega_{14}(s, \xi) \Phi_{2}(\xi) e_{A}\left(\xi, t_{0}\right) \Delta \xi \Delta s, \\
& A(t)=2^{-5 / 4} m^{-3 / 8} l_{23}(t) \int_{t_{0}}^{t} \omega_{24}(t, \xi) \Delta \xi \\
& +2^{-3 / 2} m^{-1 / 4} l_{22}(t), \\
& \Phi_{1}(\mathrm{t})=\left|C_{1}\right|+2^{-1 / 3} m^{1 / 6} \int_{t_{0}}^{t} l_{11}(s) \Delta s \\
& \quad+2^{-1 / 2} m^{1 / 4} \int_{t_{0}}^{t} l_{12}(s) \Delta s+\int_{t_{0}}^{t} l_{13}(s) \\
& \quad \cdot \int_{t_{0}}^{s}\left(2^{-1 / 2} m^{1 / 4} \omega_{13}(s, \xi)+2^{-1 / 4} m^{1 / 8} \omega_{14}(s, \xi)\right) \\
& \cdot \Delta \xi \Delta s,
\end{aligned}
$$

$$
\begin{aligned}
& \Phi_{2}(t)=\left|C_{2}\right|+2^{-1 / 3} m^{1 / 6} \int_{t_{0}}^{t} l_{21}(s) \Delta s \\
& +2^{-1 / 2} m^{1 / 4} \int_{t_{0}}^{t} l_{22}(s) \Delta s+\int_{t_{0}}^{t} l_{23}(s) \\
& \cdot \int_{t_{0}}^{s}\left(2^{-1 / 2} m^{1 / 4} \omega_{23}(s, \xi)+2^{-1 / 4} m^{1 / 8} \omega_{24}(s, \xi)\right) \\
& \cdot \Delta \xi \Delta s \\
& K(t)=\frac{1}{3} 2^{-1 / 3} m^{-5 / 6} l_{11}(t)+2^{-3 / 2} m^{-3 / 4} l_{12}(t) h_{2}(t) \\
& \cdot e_{A}\left(t, t_{0}\right)+l_{13}(t) \int_{t_{0}}^{t}\left(2^{-3 / 2} m^{-3 / 4} \omega_{13}(t, \xi)\right. \\
& \left.+2^{-9 / 4} m^{-7 / 8} \omega_{14}(t, \xi) h_{2}(\xi) e_{A}\left(\xi, t_{0}\right)\right) \Delta \xi \\
& h_{2}(t)=\frac{1}{3} 2^{-1 / 3} m^{-5 / 6} \int_{t_{0}}^{t} l_{21}(s) \Delta s \\
& +2^{-3 / 2} m^{-3 / 4} \int_{t_{0}}^{t} l_{23}(s) \int_{t_{0}}^{s} \omega_{23}(s, \xi) \Delta \xi \Delta s .
\end{aligned}
$$

In fact, the solution of system (1) satisfies the following integral equation

$$
\begin{gathered}
x^{2}(t)=x^{2}\left(t_{0}\right)+\int_{t_{0}}^{t} G_{1}\left(s, x\left(\tau_{1}(s)\right), y\left(\tau_{1}(s)\right),\right. \\
\left.\int_{t_{0}}^{s} H_{1}\left(s, \xi, x\left(\tau_{2}(\xi)\right), y\left(\tau_{2}(\xi)\right)\right) \Delta \xi\right) \Delta s, \\
y^{2}(t)=y^{2}\left(t_{0}\right)+\int_{t_{0}}^{t} G_{2}\left(s, x\left(\tau_{1}(s)\right), y\left(\tau_{1}(s)\right),\right. \\
\left.\int_{t_{0}}^{s} H_{2}\left(s, \xi, x\left(\tau_{2}(\xi)\right), y\left(\tau_{2}(\xi)\right)\right) \Delta \xi\right) \Delta s .
\end{gathered}
$$

Therefore,

$$
\begin{aligned}
& |x(t)|^{2} \leq\left|C_{1}\right|+\int_{t_{0}}^{t} l_{11}(s)\left|x\left(\tau_{1}(s)\right)\right|^{1 / 3} \Delta s+\int_{t_{0}}^{t} l_{12}(s) \\
& \cdot\left|y\left(\tau_{1}(s)\right)\right|^{1 / 2} \Delta s+\int_{t_{0}}^{t} l_{13}(s) \\
& \cdot \int_{t_{0}}^{s}\left(\omega_{13}(s, \xi)\left|x\left(\tau_{2}(\xi)\right)\right|^{1 / 2}\right. \\
& \left.\quad+\omega_{14}(s, \xi)\left|y\left(\tau_{2}(\xi)\right)\right|^{1 / 4}\right) \Delta \xi \Delta s, \\
& |y(t)|^{2} \leq\left|C_{2}\right|+\int_{t_{0}}^{t} l_{21}(s)\left|x\left(\tau_{1}(s)\right)\right|^{1 / 3} \Delta s+\int_{t_{0}}^{t} l_{22}(s)
\end{aligned}
$$




$$
\begin{aligned}
& \cdot\left|y\left(\tau_{1}(s)\right)\right|^{1 / 2} \Delta s+\int_{t_{0}}^{t} l_{23}(s) \\
& \cdot \int_{t_{0}}^{s}\left(\omega_{23}(s, \xi)\left|x\left(\tau_{2}(\xi)\right)\right|^{1 / 2}\right. \\
& \left.+\left.\omega_{24}(s, \xi) y\left(\tau_{2}(\xi)\right)\right|^{1 / 4}\right) \Delta \xi \Delta s .
\end{aligned}
$$

We can choose

$$
\begin{aligned}
& N_{11}(t, x)=x^{1 / 3}, \\
& N_{12}(t, x)=x^{1 / 2}, \\
& N_{13}(t, x)=x^{1 / 2}, \\
& N_{14}(t, x)=x^{1 / 4}
\end{aligned}
$$

and

$$
\begin{aligned}
& N_{21}(t, x)=x^{1 / 3}, \\
& N_{22}(t, x)=x^{1 / 2}, \\
& N_{23}(t, x)=x^{1 / 2}, \\
& N_{24}(t, x)=x^{1 / 4} .
\end{aligned}
$$

Since

$$
\begin{aligned}
& N_{11}(t, x)-N_{11}(t, y)=x^{1 / 3}-y^{1 / 3} \\
& \quad \leq \frac{1}{3} y^{-2 / 3}(x-y),
\end{aligned}
$$

$M_{11}(t, y)$ can be selected as $y^{-2 / 3} / 3$. Similarly, we choose

$$
\begin{aligned}
& M_{12}(t, y)=\frac{1}{2} y^{-1 / 2}, \\
& M_{13}(t, y)=\frac{1}{2} y^{-1 / 2}, \\
& M_{14}(t, y)=\frac{1}{4} y^{-3 / 4}, \\
& M_{21}(t, y)=\frac{1}{3} y^{-2 / 3}, \\
& M_{23}(t, y)=\frac{1}{2} y^{-1 / 2} .
\end{aligned}
$$

Applying Theorem 4 to (42) yields (40).

\section{Data Availability}

No data were used to support this study.

\section{Conflicts of Interest}

The authors declare that they have no conflicts of interest.

\section{Authors' Contributions}

Both the authors contributed equally to this work. They both read and approved the final version of the manuscript.

\section{Acknowledgments}

This work was supported by the National Natural Science Foundation of China (Grant nos. 61807015, 61473133), the Natural Science Foundation of Shandong Province (Grant no. ZR2017LF012), A Project of Shandong Province Higher Educational Science and Technology Program (Grant no. J17KA157), and the Doctoral Scientific Research Foundation of University of Jinan (Grant no. 1008398).

\section{References}

[1] S. Hilger, "Analysis on measure chains: a unified approach to continuous and discrete calculus," Results in Mathematics, vol. 18, no. 1-2, pp. 18-56, 1990.

[2] M. Bohner and A. Peterson, Dynamic Equations on Time Scales: An Introduction with Applications, Birkhauser, Boston, Mass, USA, 2001.

[3] M. Bohner and A. Peterson, Dynamic Equations on Time Scales: An Introduction with Applications, Birkhauser, Boston, Mass, USA, 2003.

[4] F. C. Jiang and F. W. Meng, "Explicit bounds on some new nonlinear integral inequalities with delay," Journal of Computational and Applied Mathematics, vol. 205, no. 1, pp. 479-486, 2007.

[5] A. Boudeliou, "On certain new nonlinear retarded integral inequalities in two independent variables and applications," Applied Mathematics and Computation, vol. 335, pp. 103-111, 2018.

[6] J. Wang, F. Meng, and J. Gu, "Estimates on some power nonlinear Volterra-Fredholm type dynamic integral inequalities on time scales," Advances in Difference Equations, Paper No. 257, 16 pages, 2017.

[7] Y. Tian, M. Fan, and F. Meng, "A generalization of retarded integral inequalities in two independent variables and their applications," Applied Mathematics and Computation, vol. 221, pp. 239-248, 2013.

[8] Y. Tian, Y. Cai, L. Li, and T. Li, "Some dynamic integral inequalities with mixed nonlinearities on time scales," Journal of Inequalities and Applications, vol. 2015, article 12, pp. 1-10, 2015.

[9] Y. Tian, A. A. El-Deeb, and F. Meng, "Some nonlinear delay Volterra-Fredholm type dynamic integral inequalities on time scales," Discrete Dynamics in Nature and Society, Art. ID 5841985, 8 pages, 2018.

[10] Q. H. Ma and J. Pečarić, "The bounds on the solutions of certain two-dimensional delay dynamic systems on time scales," Computers \& Mathematics with Applications. An International Journal, vol. 61, no. 8, pp. 2158-2163, 2011.

[11] S. H. Saker, "Applications of Opial inequalities on time scales on dynamic equations with damping terms," Mathematical and Computer Modelling, vol. 58, no. 11-12, pp. 1777-1790, 2013.

[12] R. Xu, F. Meng, and C. Song, "On some integral inequalities on time scales and their applications," Journal of Inequalities and Applications, vol. 2010, article 464976, 2010.

[13] T. Wang and R. Xu, "Some integral inequalities in two independent variables on time scales," Journal of Mathematical Inequalities, vol. 6, no. 1, pp. 107-118, 2012. 
[14] R. Xu and Y. Zhang, "Generalized Gronwall fractional summation inequalities and their applications," Journal of Inequalities and Applications, vol. 2015, no. 42, 2015.

[15] Q. Ma, J. Wang, R. Wang, and X. Ke, "Study on some qualitative properties for solutions of a certain two-dimensional fractional differential system with Hadamard derivative," Applied Mathematics Letters, vol. 36, pp. 7-13, 2014.

[16] Q. Ma, R. Wang, J. Wang, and Y. Ma, "Qualitative analysis for solutions of a certain more generalized two-dimensional fractional differential system with Hadamard derivative," Applied Mathematics and Computation, vol. 257, pp. 436-445, 2015.

[17] H. Liu, "A class of retarded Volterra-Fredholm type integral inequalities on time scales and their applications," Journal of Inequalities and Applications, Paper No. 293, 15 pages, 2017.

[18] H. Liu and F. Meng, "Nonlinear retarded integral inequalities on time scales and their applications," Journal of Mathematical Inequalities, vol. 12, no. 1, pp. 219-234, 2018.

[19] J. Gu and F. Meng, "Some new nonlinear Volterra-Fredholm type dynamic integral inequalities on time scales," Applied Mathematics and Computation, vol. 245, pp. 235-242, 2014.

[20] R. Xu, "Some new nonlinear weakly singular integral inequalities and their applications," Journal of Mathematical Inequalities, vol. 11, no. 4, pp. 1007-1018, 2017.

[21] R. P. Agarwal, S. Deng, and W. Zhang, "Generalization of a retarded Gronwall-like inequality and its applications," Applied Mathematics and Computation, vol. 165, no. 3, pp. 599-612, 2005.

[22] F. Meng and J. Shao, "Some new Volterra-Fredholm type dynamic integral inequalities on time scales," Applied Mathematics and Computation, vol. 223, pp. 444-451, 2013.

[23] W.-S. Wang, X. Zhou, and Z. Guo, "Some new retarded nonlinear integral inequalities and their applications in differential-integral equations," Applied Mathematics and Computation, vol. 218, no. 21, pp. 10726-10736, 2012. 


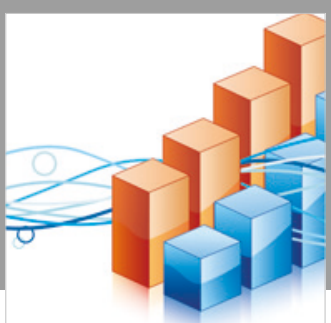

Advances in

Operations Research

\section{-n-m}
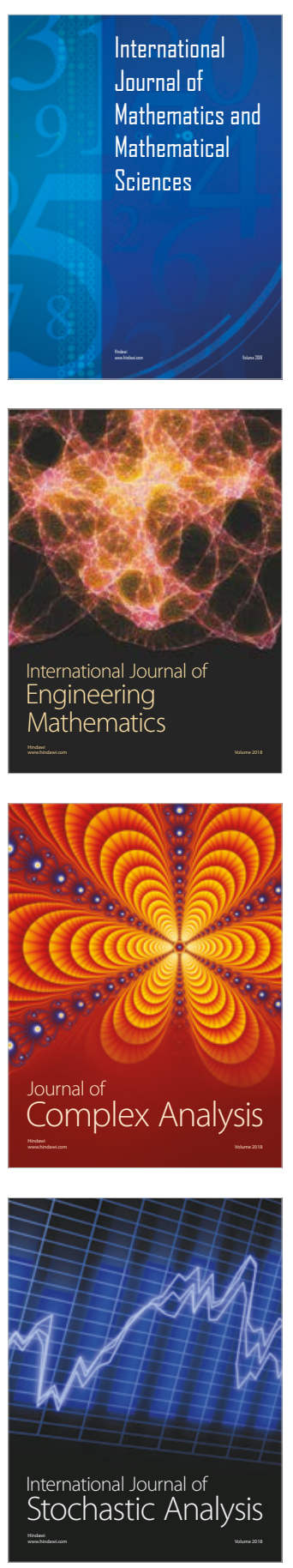
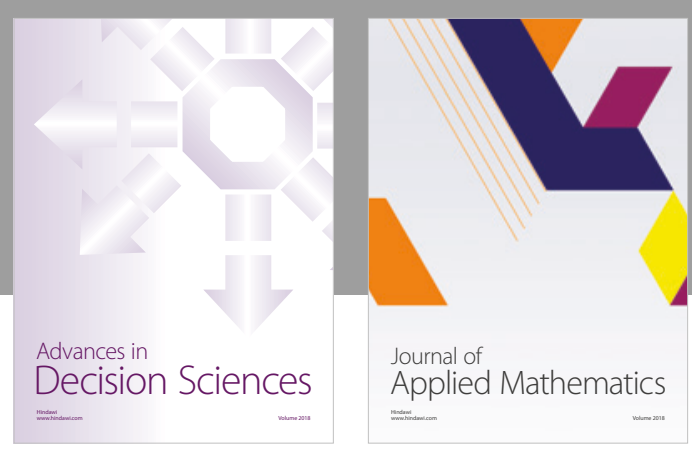

Journal of

Applied Mathematics
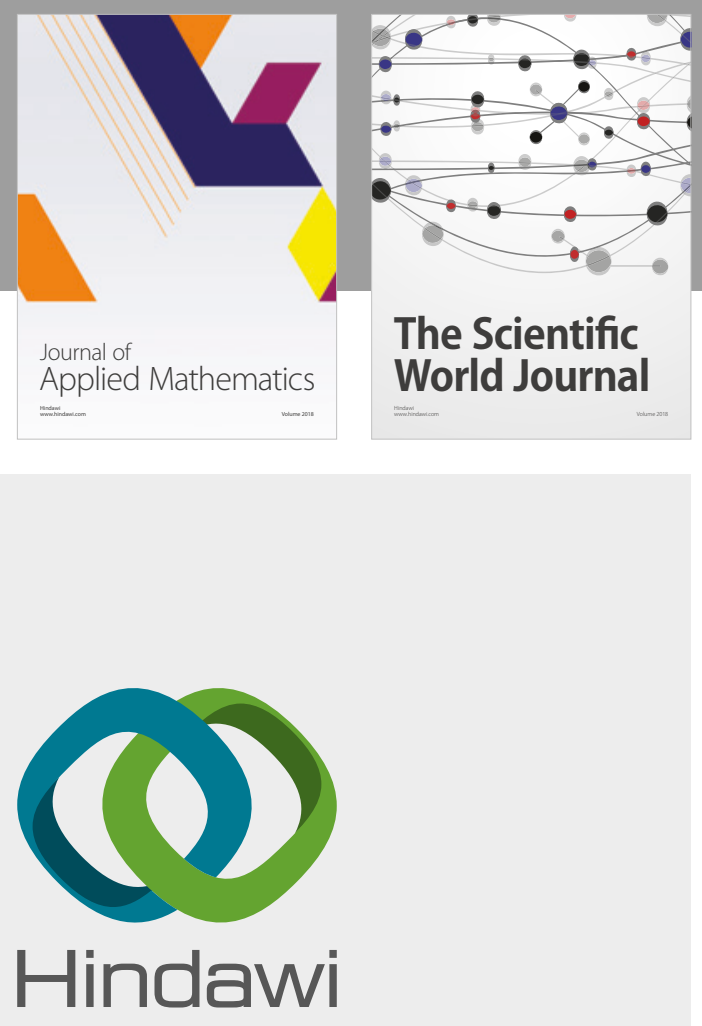

Submit your manuscripts at

www.hindawi.com

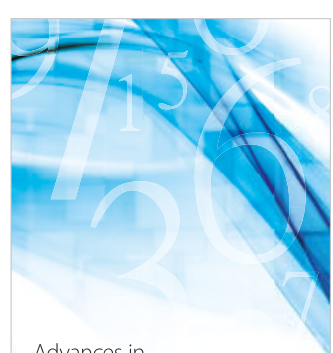

Advances in
Numerical Analysis
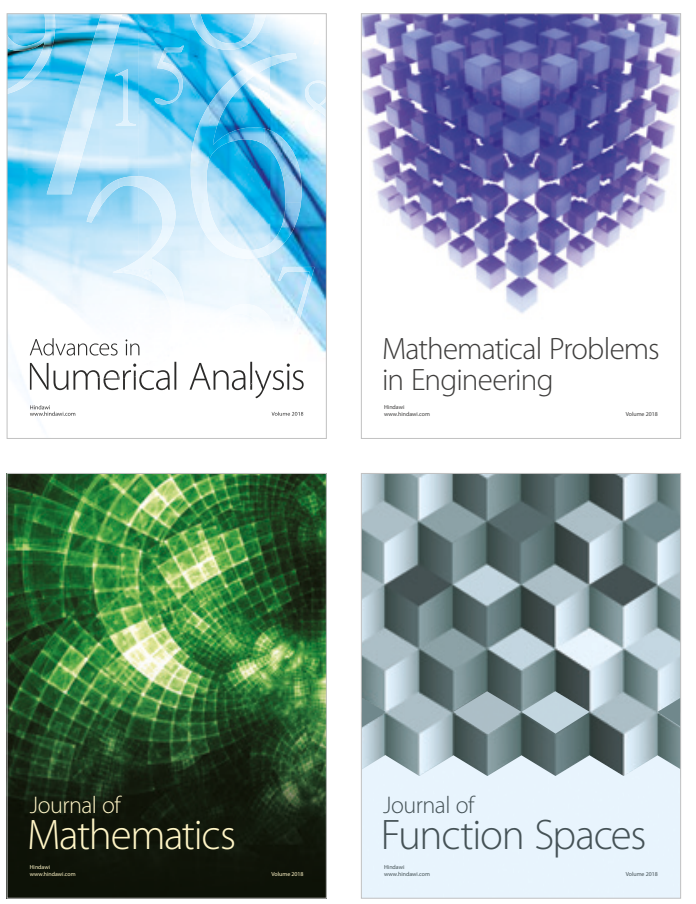

Mathematical Problems in Engineering

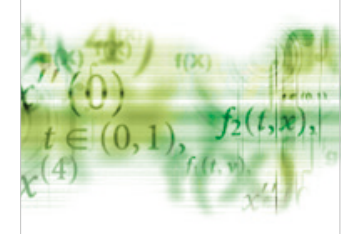

International Journal of

Differential Equations

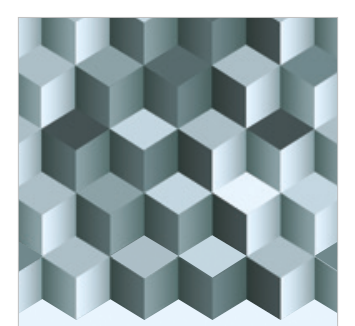

Journal of

Function Spaces

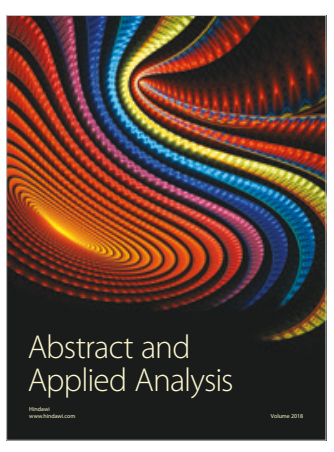

The Scientific

World Journal

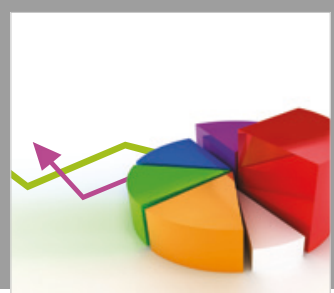

Journal of

Probability and Statistics
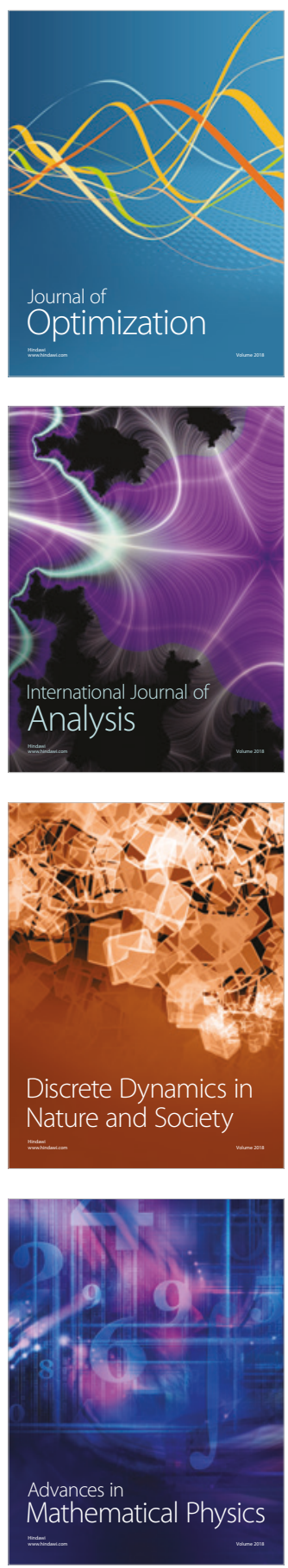